\title{
Indicadores para medir la movilidad sostenible en espacios urbanos de la sierra ecuatoriana. Caso de estudio el cantón Azogues
}

Indicators to measure sustainable mobility in urban spaces in the Ecuadorian highlands. Case study the canton Azogues

Mónica Piedad León Andrade. ${ }^{1}$ \& Viviana Veronica Carriel Bustamante. ${ }^{2}$

Recibido: 29-01-2021 / Revisado: 06-02-2021 /Aceptado: 28-02-2021/ Publicado: 05-03-2021

\begin{abstract}
. https://doi.org/10.33262/concienciadigital.v4i1.2.1738

Introduction. Sustainable mobility refers to the origin-destination movements that exist in cities, whether through public transport, motor vehicles, private vehicles, bicycles, etc., providing an optimal quality of life for the population and the environment. Objective. The present investigative work has the general objective of finding some Indicators to Measure Sustainable Mobility in Urban Spaces of the Ecuadorian Sierra, using the Canton Azogues as a case study. Methodology. For which, various theories about sustainable mobility in urban spaces and its forms of measurement at the international level were identified. Then, diagnose mobility in the urban space that comprises the Canton Azogues, to finally design the indicators. In the development of the research, theoretical and empirical methods are used such as: analysis and synthesis, the deductive and inductive method and the survey. The analysis and synthesis will be used to analyze in depth the object of study. The deductive and inductive method will help us to understand the theoretical generalities regarding sustainable urban mobility and its forms of measurement. And finally, the survey will help as a procedure in data collection through
\end{abstract}

\footnotetext{
${ }^{1}$ Mónica Piedad León Andrade, Universidad Católica de Cuenca, Facultad de Economía, Maestría en Desarrollo Local mención en Planificación, Desarrollo y Ordenamiento Territorial, Cuenca, Ecuador. monicaleon72@hotmail.com. 0000-0001-8933-5218

${ }^{2}$ Universidad Católica de Cuenca, Ecuador, viviana.carriel@ucacue.edu.ec, https://orcid.org/0000-00016572-0648
} 
a previously designed questionnaire. Results. It is evident that the majority of people travel to other places for work reasons $(61.7 \%)$ and only a minimum percentage (2\%) remain at home without moving. Conclusion. The application of these indicators in the case of Canton Azogues, is the first step for the evaluation of the different areas of sustainability in mobility issues, with which the implementation of new policies that improve social, economic and environmental aspects is intended. from the city.

Keywords: Sustainable urban mobility, mobility indicators.

\section{Resumen.}

Introducción. La movilidad sostenible se refiere a los desplazamientos origen-destino que existen en las ciudades, ya sea a través de transporte público, motorizados, vehículos privados, bicicletas, etc., brindando una calidad de vida óptima para la población y el medioambiente. Objetivo. El presente trabajo investigativo, tiene como objetivo general, encontrar algunos Indicadores para Medir la Movilidad Sostenible en Espacios Urbanos de la Sierra Ecuatoriana, utilizando como caso de estudio el Cantón Azogues. Metodología. Para lo cual, se identificaron diversas teorías sobre la movilidad sostenible en espacios urbanos y sus formas de medición a nivel internacional. Luego, diagnosticar la movilidad en el espacio urbano que comprende el Cantón Azogues, para finalmente diseñar los indicadores. En el desarrollo de la investigación se utiliza métodos teóricos y empíricos como: el análisis y la síntesis, el método deductivo e inductivo y la encuesta. El análisis y síntesis de utilizará para analizar a profundidad el objeto de estudio. El método deductivo e inductivo nos ayudará a comprender las generalidades teóricas en cuanto a la movilidad urbana sostenible y sus formas de medición. Y por último la encuesta ayudará como un procedimiento en la recopilación de datos mediante un cuestionario previamente diseñado. Resultados. Se evidencia que la mayoría de personas se transporta a otros lugares por motivos de trabajo (61.7\%) y solo un mínimo porcentaje (2\%) permanece en casa sin movilizarse. Conclusión. La aplicación de estos indicadores en el caso del Cantón Azogues, es el primer paso para la evaluación de los diferentes ámbitos de la sostenibilidad en temas de movilidad, con lo que se pretende la implementación de nuevas políticas que mejoren los aspectos sociales, económicos y ambientales de la ciudad.

Palabras claves: Movilidad urbana sostenible, indicadores de movilidad.

\section{Introducción.}

En América Latina, con el transcurso del tiempo, varias ciudades se han visto afectadas tras los profundos cambios sociales, económicos y tecnológicos que inciden notoriamente en un crecimiento urbano y económico descontrolado. Mismos que a su vez, acarrean serios problemas relacionados con la sostenibilidad y la movilidad urbana que se orientan 
más allá de la congestión, la mala comunicación, como los medios de comunicación deducen, sino tiene que ver con una negativa repercusión en la calidad de vida de las personas habitantes en estas ciudades (Ecologistas en Acción, 2017).

Es así que, las diferentes transiciones que surgen en las ciudades de acuerdo a sus características, sean estas: institucionales, sociales y económicas, centran su trabajo en desarrollar un nuevo modelo de movilidad urbana. Globalmente, este modelo tiende a incorporarse y se determina por los cambios en las razones por desplazamientos, el aumento de las distancias medias recorridas y las reformas en la localización de las actividades productivas. Sin embargo, los efectos de estos cambios de movilidad humana tienen un impacto más fuerte en países menos desarrollados (Miralles-Guasch \& Cebollada, 2009).

Por otra parte, con la tecnología se ha impulsado sistemas de conectividad que ayudan al desarrollo de servicios de movilidad basados en el uso de vehículos compartidos. Así, el abanico de posibilidades de movilidad se amplía a diario, con ello van apareciendo iniciativas para agruparlas todas bajo una misma plataforma de movilidad. La convergencia de este fenómeno con el desarrollo del vehículo eléctrico, hacen que la movilidad sea un sector que vive su momento de cambio más histórico (Torres, 2015).

Así en países como Ecuador, el Ministerio de Transporte y Obras Públicas (MTOP, 2020) refiere que a escala nacional el $82 \%$ de personas se traslada en transporte público, $11 \%$ usa vehículo particular, $3 \%$ taxi, $2 \%$ camina, 1,2\% maneja bicicleta, y el 0,8\% utiliza otras opciones. Por lo que, al margen de análisis de la situación de movilidad, se han implementado políticas para enfrentar los problemas antes descritos, entre las que se resalta la propuesta de Plan Nacional de Movilidad Urbana Sostenible de Ecuador.

En este contexto, los Gobiernos Autónomos Descentralizados (GAD) se encargan netamente del control, regulación y planificación del tránsito, transporte terrestre y sobre todo la seguridad vial en territorio, con: acceso a las mismas oportunidades, descartar el uso de transporte no motorizado, mejorar el trabajo del transporte público e incrementar su uso, planificación de uso de suelo y movilidad urbana, estímulos económicos y políticas de estacionamiento (MTOP, 2020).

Ahora bien, para esta investigación es importante conocer los datos estadísticos poblacionales de la ciudad de Azogues, en la cual se evidencia un incremento humano en la zona urbana ya que, para el año 1950 la población urbana era del 13\% del total de la población y la rural $87 \%$. Mientras que, el último censo que realizó el Instituto Nacional de Estadísticas y Censos (INEC, 2010) la población urbana fue un $48 \%$ y la población rural un 52\% lo que significa que Azogues tenía 39.908 habitantes. Para el año 2020, según los análisis realizados por Pérez Cotrina (2017) se estima que la población en la ciudad sería de 46.787 habitantes con mayor concentración en la zona urbana. 
El GAD Municipal de Azogues (GADMA, 2013) efectuó el Plan de Transporte y Seguridad Vial para el Cantón, en el cual sus estudios determinaron redes de transporte urbano e interparroquial, redes viales, componentes técnicos para administración del transporte y determinación de políticas institucionales para su ejecución y manejo. Posteriormente, esta institución asumió las competencias de control y regulación del tránsito en la ciudad.

Tras un análisis del plan de movilidad de la Ciudad de Azogues, se pueden identificar las problemáticas a ser sujetas de estudio en la presente investigación, básicamente, temas relacionados con sostenibilidad ambiental, social y energética. Así también, el incremento de tiempos y costos de traslado para los habitantes que se desplazan cotidianamente, transgrediendo tanto en la actividad económica poblacional como en el deterioro del medio ambiente de la ciudad.

\section{Fundamentación Teórica}

El presente trabajo de investigación toma en consideración que la ciudad debe ser estudiada desde diferentes ángulos, teniendo siempre presente al ser humano como su principal protagonista. De este enfoque surge como idea central que la ciudad puede ser conceptualizada como el lugar desarrollado por los hombres para vivir juntos, resolver sus necesidades y optimizar sus contextos de vida. En este sentido, se realiza un estudio comparativo de dos modelos de desarrollo urbano antagónicos que son la ciudad compacta y la ciudad dispersa. En la que desataca, la ciudad compacta como el modelo urbano más conveniente, ya que esta no debe ser solo un "espacio para vivir" sino un "espacio para convivir" considerando la productividad urbana, inclusión social y medioambiente sostenible, las dimensiones que configuran la sostenibilidad (Alarcón, 2020).

Con el transcurso del tiempo se han desarrollado propuestas por mejorar la movilidad, mismas que se enfocan fundamentalmente en la velocidad de desplazamientos cotidianos basados en el aumento de la capacidad vial, derivando de esta manera, en el incremento del parque vehicular y la aparición de nuevas demandas. Por lo tanto, se debe pensar en ciudades más incluyentes que no solo busquen la eficiencia en los traslados y políticas de transporte más sustentables, sino también brinden una justa accesibilidad a las diferentes oportunidades que oferta la ciudad (Gakenheimer, 1998).

Para una mayor comprensión del tema de estudio, es importante entender la definición de movilidad. Mendoza (2017) afirma que:

La movilidad se conceptualiza en los desplazamientos origen-destino que tienen lugar en las ciudades, ya sea por medios de transporte motorizados o no motorizados, particulares o colectivos, haciendo referencia a la clasificación general de los modos de transporte que una persona puede utilizar para trasladarse de un lugar a otro. (p.1) 
Así también, Flores Juca et al. (2017) refiere que "la movilidad urbana es un parámetro que mide la cantidad de desplazamientos realizados por personas o mercancías en un ámbito social" (p. 14), por lo tanto, es entendida como la necesidad o el deseo de una población de desplazarse. Además, es un derecho social para la movilización de todos los ciudadanos de un territorio por lo que es una obligación de sus gobiernos preservar y garantizar de forma igualitaria brindando óptimas condiciones dentro del sector urbano e interurbano de la ciudad (Valladolid, 2005, como se citó en Dirección General de Industria, Energía y Minas de la Comunidad de Madrid, 2010).

Dentro de las economías modernas resulta indispensable en el sistema de transporte que logre un equilibrio entre la accesibilidad y las necesidades de movilidad para una población. Sin embargo, el aumento de los ingresos per cápita ha generado un uso expansivo de autos particulares como medio de transporte de pasajeros en áreas urbanas, lo que se convierte en un factor de riesgo para la sostenibilidad ambiental. Por esta razón, centrar estudio y no escatimar esfuerzos por una movilidad de calidad en la cual los ciudadanos pueden disfrutar de un espacio con desplazamientos seguros y, además, minimizar costos de tiempo y energía (Lizárraga, 2006; Dirección General de Industria, Energía y Minas de la Comunidad de Madrid, 2010).

En la actualidad, el transporte urbano ocupa un papel esencial dentro del ámbito económico y social de las ciudades, pues la dispersión urbana influye directamente en la demanda social de movilidad. De ahí la importancia de nuevas perspectivas de movilidad urbana en cuanto a formas de organización del trabajo y transformación productiva, las cuales deben abarcar también desde un ámbito de sostenibilidad medioambiental hasta la competitividad de una ciudad. Estos criterios se contemplan como objetivos principales de estudio en relación a temas de ordenamiento territorial (Falcón Suárez et al., 2016; García Schilardi, 2017).

\section{Movilidad Sostenible}

Desde un punto de vista de movilidad sostenible, se plantea como aquella ciudad que se construye así misma en base a principios ecológicos, educadores y de equidad; es decir, se caracteriza por: una movilidad efectiva, ahorro de energía y recursos hídricos, disminución de la polución auditiva y construcción de áreas verdes en lugares públicos mismos que sean recreativos y agradables para la población. De este concepto nace la ciudad sostenible perfecta, basada en autoabastecimiento energético y que aprovecha sus residuos como nuevas materias primas (Díaz-Contreras \& Díaz-Vidal, 2019).

La movilidad sostenible implica una interdependencia entre transporte y movilidad, en donde mejorar el transporte es efectivizar la movilidad y por ende el bienestar y calidad de vida de una población. Es decir, es un modelo que no ocasiona un impacto negativo ambiental y al contrario, aprovecha las alternativas de la movilidad sin sacrificar el entorno y agotar recursos de las próximas generaciones (Flores Juca et al., 2017; Zuluaga, 2017). 
Para los principales actores de la movilidad sostenible sus intereses se centran en patrones de transporte, tránsito e infraestructura vial, así como en la implementación de formas de un desplazamiento llevadero (caminar, bicicleta y transporte público) dentro de una ciudad. Con la única finalidad de cubrir las necesidades medioambientales, económicas, sociales y garantizar espacios seguros, concurrentes con la salud de una población y ecosistemas (Lizárraga, 2006; Universidad Politécnica de Valencia [UPV], 2012).

Supremamente, estudiar el concepto de movilidad es ahondar en los elementos o indicadores que dan sostenibilidad a la misma, es decir, enfocarse en un sistema capaz de reducir la cantidad de vehículos que circulan por las vías, así como responder a múltiples variables como la contaminación, el ruido, tarifas, espacios de recreación, entre otros (Guillamón \& Hoyos, 2005).

\section{Indicadores de medición de la movilidad sostenible en espacios urbanos}

En la actualidad, los indicadores que nos permiten medir la eficacia de los sistemas de transporte carecen de importancia en los estudios de movilidad, pese a ser un componente fundamental en la dinámica de las ciudades. Por lo que, es indispensable incluir indicadores en cualquier proceso de elaboración de un estudio, proyecto o actividad de desarrollo sostenible, ya que, permite cuantificar o comparar resultados para la implementación o no de las políticas de movilidad sostenible de acorde a las necesidades de una ciudad. Además, el propósito de los indicadores es evaluar, controlar e informar al público sobre la evolución de los factores sociales, medioambientales y económicos, así como de las actividades accionadas en pro del ambiente y población beneficiada (Motos, 2019, p. 34-35).

En este contexto, Quiroga (2009) refiere la diferencia entre indicador ambiental e indicador de desarrollo sostenible. Por un lado, el indicador ambiental se encarga de identificar y poner en conocimientos los estados y las primordiales dinámicas ambientales; $y$, por otro, los indicadores de desarrollo sostenible pretenden dinamizar las medidas económicas, sociales y ambientales y sus interrelaciones. Los indicadores, como tal, son una buena fuente de recursos, así como también una herramienta para concentrar esfuerzos en la elaboración de información seleccionada, priorizando aquella que sea clave para tomar decisiones eficientes (p. 21-22).

Existen diferentes maneras para elegir y construir indicadores. Gaviria (2013) propone la identificación, priorización y selección de indicadores de sostenibilidad. Para ello, se inicia por la revisión de documentación, recopilación de información a través de entrevistas o encuestas, lluvia de ideas, comparación con otras áreas y herramientas existentes; además, análisis de la base de información histórica y el conocimiento adquirido a través de proyectos similares anteriores. Por otro lado, Jiménez (2008) señala en su estudio, diez pasos para construir indicadores: 
Establecer las definiciones estratégicas, Establecer los aspectos relevantes a medir, Formular los indicadores, Validar los indicadores, Recopilar la información, Establecer metas y periodicidad de medición, Establecer la fuente de información, Establecer supuestos, Evaluar los resultados, Informar los resultados y la evaluación. (diapositiva 22)

Así también, Rodríguez-Chávez (2016) analiza una serie de indicadores de la movilidad sostenida como: los ámbitos del transporte, el medio ambiente, el desarrollo social y económico, el urbanismo, el empleo y la vivienda, para divisar alternativas y estrategias de solución a los traslados diarios de la sociedad, optimización del espacio público, el consumo de recursos naturales y en este sentido, crear sistemas eficientes que den resultados inmediatos a la situación que presentan las ciudades hoy en día.

De la misma manera, otros autores establecen criterios generales para elegir indicadores apropiados: Relevancia y pertinencia, Claridad de enfoque y significado, Medibles y verificables, Disponibilidad y calidad de datos, Universales y comparables y Sensibles (Flores Juca et al., 2017). Por su parte, Motos (2019) refiere una metodología centrada en medir el estado actual de movilidad urbana y desarrollo en una población, esto, a través de: determinar un conjunto inicial de datos, usar la programación lineal para la selección de indicadores y escoger indicadores que abarcan la mayor área posible y proporcionan la máxima cantidad de información del conjunto de datos (p. 50-51).

Por último, el modelo PER-FPEIR ${ }^{3}$ que explica la Organización para la Cooperación y el Desarrollo Económicos (OCDE), busca la obtención de indicadores que describan un esquema de forma secuencial y causal (Espejel, 2009). Sin embargo, este modelo muestra una excesiva linealidad, por lo que plantea como alternativa un esquema general del territorio que implique un sistema de interrelaciones entre sus partes; es decir, un sistema de indicadores con una doble lectura vertical y horizontal. La lectura horizontal incluye niveles de interrelación en las tres dimensiones de sostenibilidad: económica, social y ambiental, mientras que, la lectura vertical sigue el esquema PER-FPEIR con una lógica de causalidad, adaptada a la visión territorial y a la visión sectorial (Urbano et al., 2011).

Entonces, para que un indicador de movilidad sostenible pueda ser implementado debe cumplir con ciertas condiciones como: la disponibilidad de los datos, ser medibles y verificables, es necesario la identificación del nivel de aplicación por tipo de proyecto, ser comparables, reproducibles y repetibles, y por supuesto, relevantes (Centro de Trasporte Sustentable EMBARQ México [CTSEMBARQ México], 2015). No obstante, hay que tomar en consideración las observaciones y evitar ciertos aspectos críticos en la medición de la movilidad sostenible que son: la proliferación de indicadores totalmente desorganizados, la desproporción en la influencia de ciertos indicadores (desequilibrio),

\footnotetext{
${ }^{3}$ PER-FPEIR: Presión (P)-Estado (E)-Respuesta (R). El modelo FPEIR (Fuerzas motrices-Presión-Estado-ImpactoRespuesta). Es decir, generar indicadores que describan: Cómo se encuentra el territorio (Estado), Cuáles son las causas que explican su condición (Presión), Cuáles son las causas mediatas que explican las presiones (Fuerzas motrices) y Cuáles son las medidas que ha tomado la sociedad para mitigar los impactos negativos (Impacto-Respuesta).
} 
indicadores seleccionados a partir del oportunismo (cuando no hay acceso a los datos) y la medición de las situaciones sociales o ambientales en base a indicadores económicos (Caniffi, 2017).

Finalmente, el presente trabajo de investigación tiene como objetivo general identificar los indicadores de sostenibilidad para el transporte urbano en el cantón de Azogues, frente al carecimiento de estudios actuales en este tema y la falta de importancia que se ha dado a los diferentes niveles que abarcan una movilidad adecuada y efectiva. Por lo que, la finalidad de este estudio es la creación de herramientas para la planificación de una movilidad sostenible que permita mejorar las situaciones sociales, económicas, ambientales y en sí, la calidad de vida de la población tanto del sector urbano como rural de la ciudad. Para ello se medirá los diferentes efectos que se producen por la movilidad urbana actual del cantón Azogues.

\section{Metodologia.}

La amplia revisión bibliográfica, muestra diversas propuestas sobre la utilización de indicadores de movilidad desarrollados en varios países del mundo, de los cuáles se han escogido los que más cercanos a la realidad actual del cantón Azogues, para posteriormente, analizar el caso de estudio a partir de los resultados obtenidos y finalmente, definir los indicadores más apropiados para medir la movilidad sostenible en espacios urbanos del lugar de estudio. De esta manera, se consideran varios autores para la creación de un sistema de indicadores.

Según Motos (2019) los agrupa en cuatro tipos: indicadores económicos, sociales, ambientales y específicos. Mientras que, Rodríguez-Chávez (2016) propone indicadores basados en el concepto de supermanzanas: decrementar la contaminación por tráfico, congestión, ruidos de toda índole, accidentes de tráfico, así como el retomar el espacio público, y, además, la edificación de sistemas eficientes que den solución a la problemática actual de una ciudad.

Una investigación realizada en la ciudad de Mendoza sobre el análisis de diversas variables e indicadores que ayudan a medir si el modelo de movilidad tiende a ser sostenible. Principalmente, se evalúa la dimensión operativa del transporte colectivo considerando variables como: estructura física de las rutas y de la red, infraestructura y equipamiento del sistema de transporte, cobertura, cobertura del área de transporte y tiempo de viaje y frecuencia (García Schilardi, 2017).

Así mismo, un estudio ejecutado en la ciudad de Cuenca se centra en la identificación de un conjunto de indicadores de sostenibilidad para el transporte rural de la ciudad, con la finalidad de convertirse en una instrumento de planificación en la que evalúan los conceptos de sostenibilidad y movilidad sostenible, su evolución histórica, para luego definir la jerarquía de los indicadores (Flores Juca et al., 2017). 
Para efecto de la investigación se contó una población de 370 personas, para el tamaño de la muestra se aplica un nivel de confianza del 95\%. En base a lo expuesto, el $62,2 \%$ de encuestados fueron hombres, $27 \%$ mujeres. El 71,7\% trabajan activamente, 18,3\% estudia, y el $10 \%$ se dedica a otras actividades. Se analizó además la información sobre vehículos. En la que se obtiene que el $90 \%$ tiene licencia de conducir, el $80 \%$ posee vehículo propio, teniendo por familia un promedio de 52\% solo 1 vehículo, el $46 \%$ dos vehículos y el $2 \%$ más de dos vehículos.

Para la recolección de datos se tomó en cuenta una zonificación de 7 distritos donde se realizó varias encuestas que ayudarán como un procedimiento en la recopilación de datos mediante un cuestionario previamente diseñado, sin modificar el entorno ni el fenómeno donde se recolecta la información a analizar, mediante contacto directo con los diferentes actores de la Ciudad, la que se considera certera y servirá para formular los indicadores de movilidad urbana sostenible.

\section{Resultados.}

\section{Análisis del estudio de caso}

\section{El cantón de Azogues}

San Francisco de Peleusí de Azogues con clima templado es la capital de la provincia del Cañar. Dividido en 9 parroquias, siendo Azogues la única Parroquia Urbana, posee 39.908 habitantes aproximadamente, según el INEC (2010). Se encuentra ubicada al sur de la Provincia del Cañar, dentro de la conurbación de Cuenca junto a los cantones de Biblián y Déleg; las dos ciudades están conectadas por la autopista Cuenca - Azogues.

Tabla 1

Proyección de la Población del Cantón Azogues

\begin{tabular}{llll}
\hline Parroquia & Año 2010 & Año 2019 & Año 2020 \\
\hline Azogues & 39808 & 46111 & 46787 \\
\hline
\end{tabular}

Nota. Esta tabla indica el número de habitantes posibles en los diferentes años. Fuente: INEC (2010).

Por otra parte, la problemática de movilidad es evidente sobre todo en la zona central del cantón Azogues, se trata del congestionamiento vehicular (3.82\% según MTOP, 2020), mismo que se debe a la utilización desorganizada de vehículos privados, transporte público, transporte colectivo (taxis y camionetas), entre otros; a esto se suma el crecimiento urbano de la población.

La problemática explicada existe porque en el centro del cantón se concentra la mayoría de actividades laborales, incrementando el número de vehículos (privados o públicos) y transeúntes desde las parroquias Charasol y Javier Loyola. El caos vehicular se agrava aún más por la configuración de las vías angostas y pendientes que induce a una mayor 
congestión en horas pico (en la mañana 7 a 8 am, al medio día de 12 a 14 pm y en la tarde de 17 a 18 pm) (Pérez Cotrina, 2017).

Además, los ciudadanos refieren que el transporte público no brinda seguridad y comodidad por lo que prefieran trasladarse en sus vehículos privados, incrementando la flota vehicular en la vía. En tanto, el transporte comercial, aumenta para suplir las necesidades de quienes usan este medio, la mayoría de personas lo utilizan para desplazarse en distancias cortas dentro del cantón (0-2Km). Finalmente, los espacios de estacionamiento para cooperativas de taxis y camionetas, en zonas urbanas, aumentaron en los últimos años lo que disminuye el estacionamiento para usuarios particulares (Pérez Cotrina, 2017).

Servicio municipal de estacionamiento rotativo tarifado de Azogues (SEMERTAZ). La creciente tasa de motorización, la alta concentración comercial, mercados tradicionales de expendio de alimentos y otros artículos variados en el núcleo central de la ciudad de Azogues, tras la búsqueda de control vial dan origen a una entidad denominada SEMERTAZ. Esta institución, busca cubrir la demanda de estacionamiento en la ciudad, orden en la circulación vehicular y peatonal, incremento de la capacidad vial para la circulación en el centro urbano, ofertar plazas de estacionamiento en los predios urbanos dentro de las zonas centrales; dinamizando, de esta manera, un consecuente desarrollo productivo (GADMA, 2013).

Tabla 2

Plazas de Estacionamiento en el Área Estudio

\begin{tabular}{lllll}
\hline & Normales & Discapacitados & Motos & Total \\
\hline Total & 862 & 97 & 97 & 1056 \\
Porcentaje & $81.63 \%$ & $9.19 \%$ & $9.19 \%$ & $100 \%$
\end{tabular}

Nota. Esta tabla indica el número de espacios para estacionar disponibles para diferentes sectores de la población. Fuente: Elaboración propia con base en datos del Plan de Transporte, Tránsito y Seguridad Vial de Azogues (GADMA, 2013).

Sistema vial. El gobierno central planifica, financia construye y mantiene la red vial principal, en tanto que los gobiernos autónomos descentralizados municipales tienen como competencia exclusiva la de planificar, controlar y regular el transporte, tránsito y seguridad vial. En Azogues, de acuerdo a la Ordenanza del uso del suelo en su art. 77 refiere, que las vías urbanas de la parroquia se clasifican en: arteriales, colectoras, locales, peatonales, peatonales con acceso vehicular restringido (Ordenanza Sustitutiva de Reglamentación del Uso de Suelos Urbano y Rural de Azogues, 2009). 
Figura 1

Plano del Sistema Vial de la Parroquia Urbana de Azogues

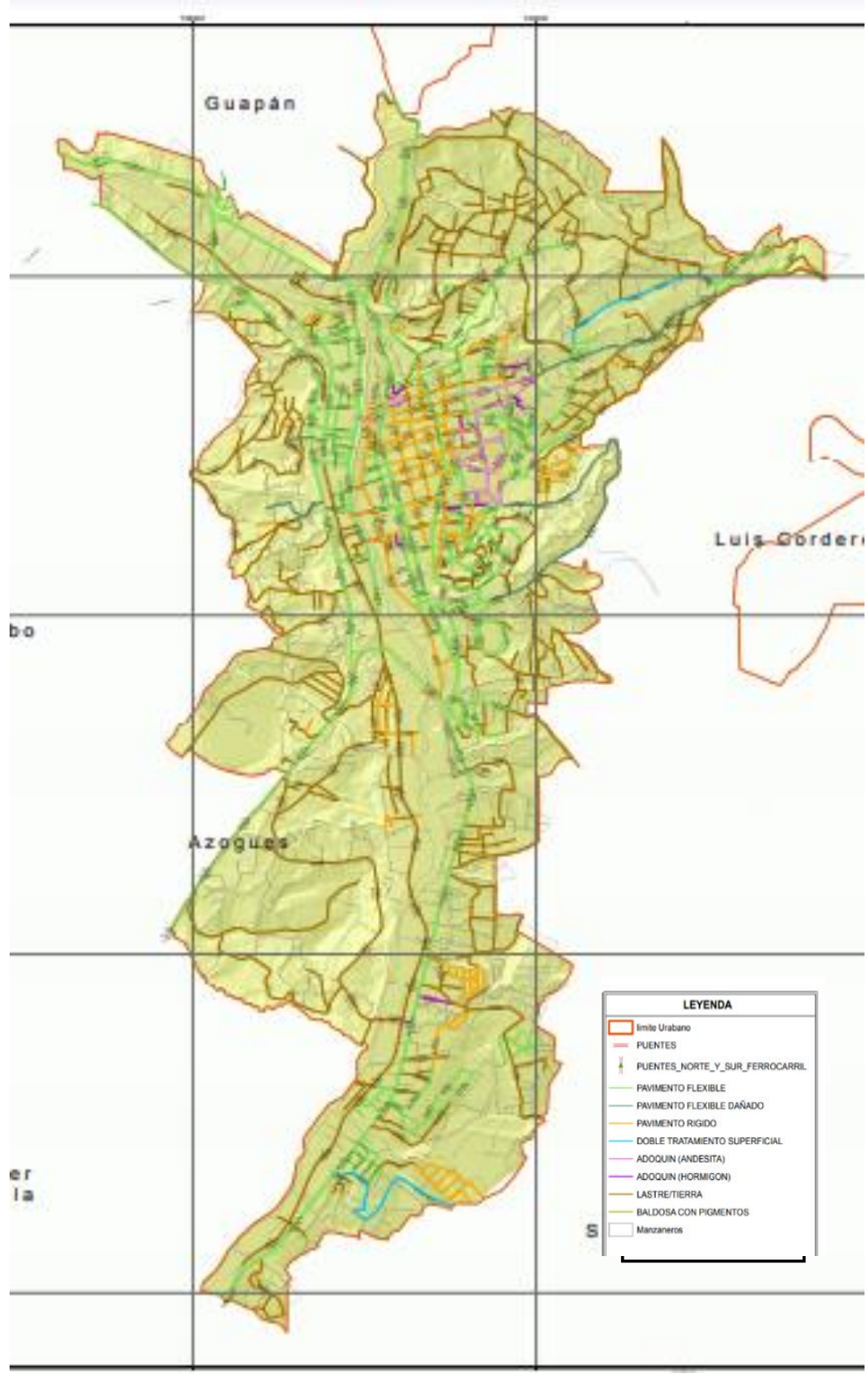

Nota. En el gráfico se observa la clasificación de las diferentes vías en la zona urbana. Tomado del Plan de Desarrollo y Ordenamiento Territorial del Cantón Azogues (FIPAD, 2020).

Tabla 3

Tipos de Vías con su Longitud en el Cantón Azogues

\begin{tabular}{lccc}
\hline & Tipo de Vía & Metros & Total \\
\hline Asfalto & & 55.741 & \\
& Bueno & 112.294 & 205.8891 \\
& Regular & 37.8541 & $(17.97)$ \\
\multirow{2}{*}{ Pavimento } & Malo & & \\
& & 122.316 & 152.2417
\end{tabular}




Malo 29.7258

Otros

$\begin{array}{ccc}\text { Bueno } & 45.1212 & \\ \text { Regular } & 741.3747 & 787.3698 \\ \text { Malo } & 0.8739 & \text { (68.73) }\end{array}$

Nota. Esta tabla refiere que el tipo de vías que existen en el Cantón Azogues: presenta asfalto con un $17.97 \%$, el pavimento el $13.29 \%$ y otros tipos de vías con el $68.73 \%$. * Longitud = Metros. Fuente: Elaboración propia con base en datos del Plan de Desarrollo y Ordenamiento Territorial del Cantón Azogues (FIPAD, 2020).

Número de vehículos matriculados. La Unidad de Matriculación del GAD Municipal de Azogues (2018) informa que cada año son más el número de vehículos, de diferentes tipos, que han sido matriculados. Lo que evidencia el notable crecimiento poblacional del cantón. Así como también, muchos de estos vehículos no son revisados técnicamente a profundidad para evitar el impacto ambiental producidos por los mismos.

Tabla 4

Diferentes Tipos de Vehículos Matriculados

\begin{tabular}{lcc}
\hline \multicolumn{1}{c}{ Tipo de Vehículo } & Número de Vehículos & Porcentaje \\
\hline Buses & 182 & $1.03 \%$ \\
Camiones & 386 & $2.18 \%$ \\
Plataformas & 22 & $0.12 \%$ \\
Taxis & 225 & $1.27 \%$ \\
Camionetas & 331 & $1.87 \%$ \\
Particulares & 15.703 & $88.79 \%$ \\
Motos & 836 & $4.73 \%$ \\
\hline Total de Vehículos & 17.685 & $100 \%$
\end{tabular}

Nota. Esta tabla indica que existe más vehículos particulares (88.79\%) y menor cantidad de vehículos de plataforma: tráiler, maquinaria pesada (0.12\%). Fuente: Elaboración propia con base en datos de la Unidad de Movilidad GADMA.

Tasa de crecimiento del parque automotor. Para realizar la proyección del parque automotor del Cantón Azogues, la Agencia Nacional de Tránsito (ANT) refiere datos de los vehículos matriculados en los años 2017 (16.148) y 2018 (17.584) acorde al domicilio, ya que no cuenta con datos completos y no reflejan la realidad de los vehículos matriculados.

Figura 2

Fórmula para determinar el porcentaje de crecimiento anual

$$
T_{c}=\frac{C V_{n+1}-C V_{n}}{C V_{n}} * 100 \%
$$

Nota. ${ }^{*} T_{c=}$ Tasa de crecimiento, $N=$ Año en el que se evalúa, $C V n=$ Cantidad de vehículos en el año $\mathrm{n}$. $C V_{n+1=}$ Cantidad de vehículos en el año $\mathrm{n}+1$. Tomado de la Dirección de Movilidad del GADMA (2017). 
Tabla 5

Tasa de Crecimiento Vehicular Anual en el Cantón Azogues

Fórmula de Tasa de Crecimiento

Total de Tasa de Crecimiento

$$
T_{c}=\frac{17.584-16.148}{16.148} * 100 \%
$$

$8.89 \%$

Nota. El análisis de la tasa de crecimiento vehicular anual corresponde a los años 2017 (16.148) al 2018 (17.584) con un incremento de $8.89 \%$ por año. Lo que significa que para el año 2019 a 2020 habría un total de 19.149 y 20.849 vehículos respectivamente. Fuente: Elaboración propia con base en datos de la Agencia Nacional de Tránsito (2017).

Polos generadores de viajes (zonas). Son zonas que donde existen el mayor o un gran número de desplazamientos dentro una ciudad, donde se generan actividades, que directa o indirectamente, demanda de usos de suelo. Sin embargo, causan efectos negativos como la inaccesibilidad y la inseguridad poniendo en riesgo las condiciones de vehículos y transeúntes (Herrera \& Jaramillo, 2006).

Figura 3

Plano de las Zonas Urbanas del Cantón Azogues

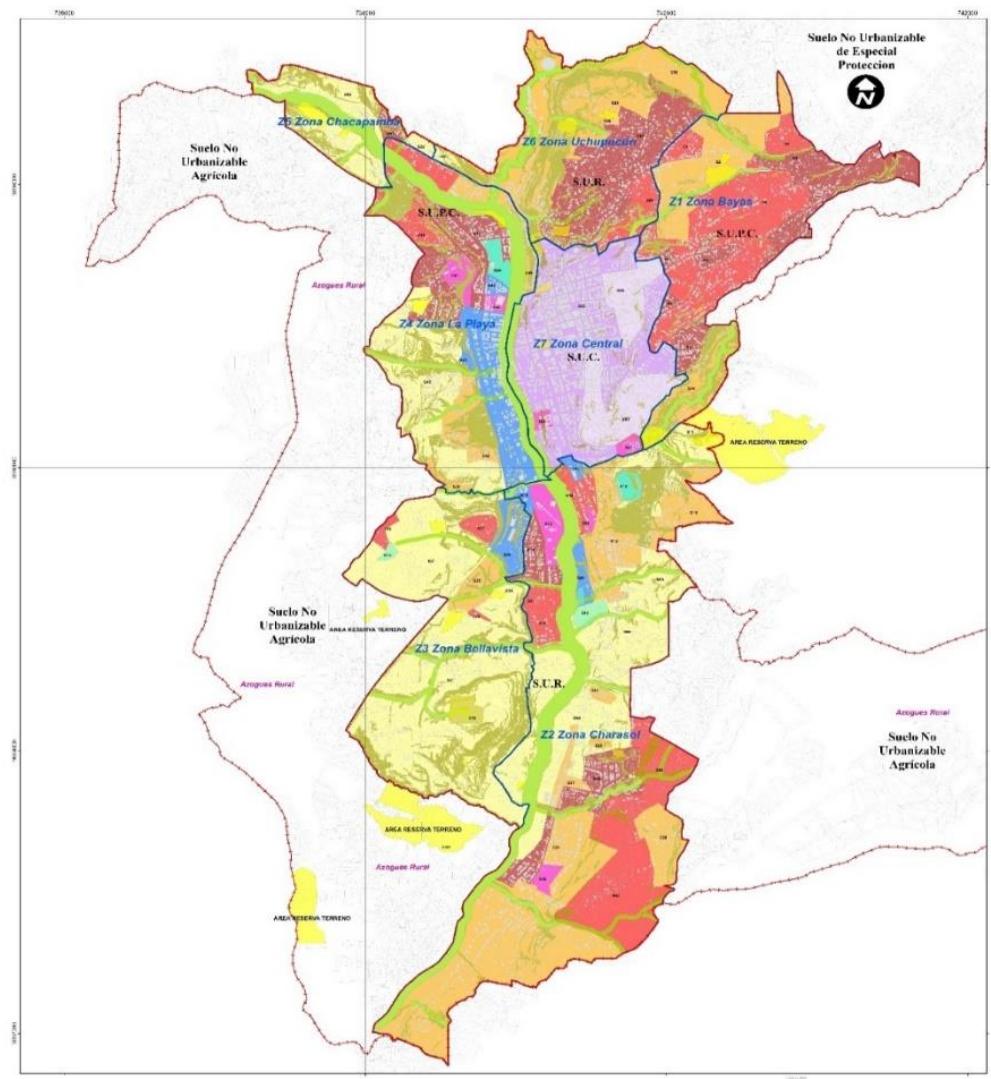

Nota. Z1 = Bayas, Z2 = Charasol, Z3 = Bellavista, Z4 = La Playa, Z5 = Chacapamba, Z6 = Uchupucún, Z7 = Central. Esta figura nos señalas la división de las 6 zonas urbanas del cantón Azogues. Fuente: Plan de Desarrollo y Ordenamiento Territorial del Cantón Azogues (FIPAD, 2020). 


\section{Transporte}

Sistema de transporte público. La Ciudad de Azogues se encuentra circulada por el sistema de transporte público en la zona urbana, la compañía TRURAZ CIA. LTDA., misma que tiene 8 líneas y 41 vehículos que brindan su servicio en toda la ciudad. La red vial recorre las vías arteriales y colectoras que están únicamente pavimentadas. Solo pocas líneas de transporte público recorren a los sitios de la ciudad que se encuentran con lastre. Además, debido a la topografía irregular de la parroquia las líneas recorren vías de pendientes elevadas (Pérez Cotrina, 2017).

En cuanto al transporte público, se obtuvo que el 76,7 \% lo usan, y apenas el 23,3\% de la población encuestada no usan el transporte público. Así mismo, el 41\% manifiestan que lo usan por problemas de aparcamiento en la ciudad y prefieren dejar sus coches en la casa, mientras que el $27 \%$ manifiesta no tener vehículo particular, otro $27 \%$ dice tener otras razones, y apenas el 5\% expone que lo usa por rapidez.

Figura 3

Número de Unidades Fabricadas por la Cooperativa TRURAZ

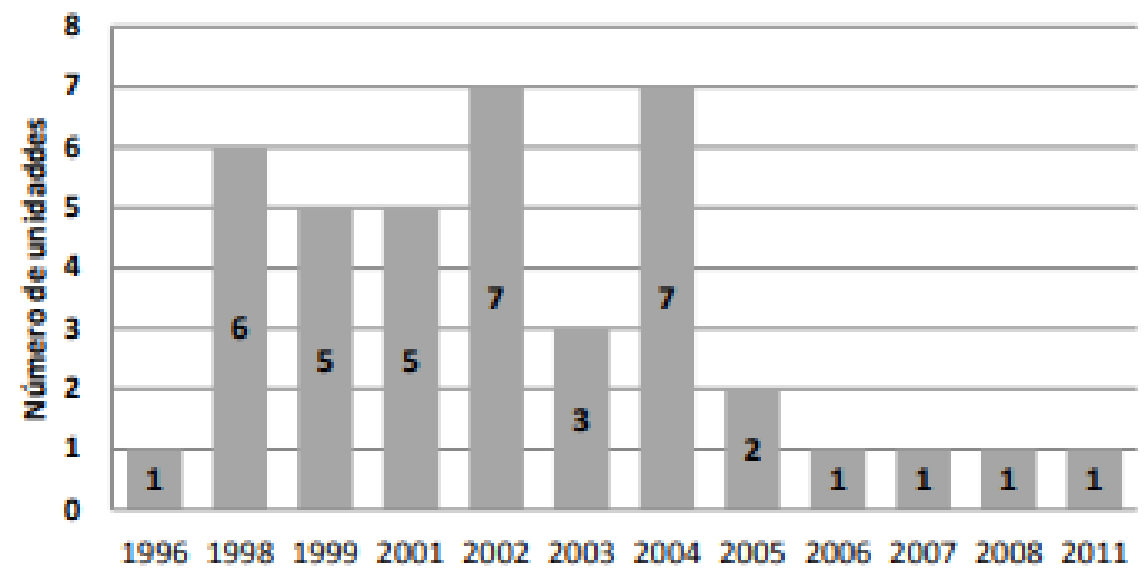

Nota. En el año 2002 y 2004 mayor número de unidades fabricadas. Tomado de Morocho \& Rodríguez (2019).

Encuestas Origen-Destino. De acuerdo al tamaño de la muestra mencionado se efectuaron encuestas origen - destino tomando como puntos de llegada y partida las diferentes zonas urbanas planteadas por la Ordenanza Sustitutiva de Reglamentación del Uso de Suelos Urbano y Rural de Azogues (2009). Las encuestas se realizaron entre la semana del 25 al 30 de octubre del 2020, se usó un modelo simplificado de encuesta Origen-Destino.

Tabla 6

Incidencia de Origen-Destino de la población desde las diferentes Zonas Urbanas

\begin{tabular}{lclc}
\hline Zonas Origen & Porcentaje & Zonas Destino & Porcentaje \\
\hline Bayas & $9.4 \%$ & Bayas & $2.3 \%$ \\
Charasol & $29.2 \%$ & Charasol & $28.6 \%$ \\
\hline
\end{tabular}




\begin{tabular}{lclc}
\hline Bellavista & $8 \%$ & Bellavista & - \\
La Playa & $15.3 \%$ & La Playa & $8.5 \%$ \\
Chacapamba & $6.2 \%$ & Chacapamba & $1.4 \%$ \\
Uchupucún & $6.4 \%$ & Uchupucún & $2.1 \%$ \\
Central & $25.5 \%$ & Central & $57.1 \%$ \\
\hline
\end{tabular}

Nota. En esta tabla se muestra que la Zona 2 y 7 existe la mayor concentración de personas que parten de esos lugares, así como también, son las Zonas a las que concurren la mayoría de la población. Fuente: Elaboración propia en base a datos obtenidos del presente estudio.

Tabla 7

Motivo de Viaje de la Población del Cantón Azogues

\begin{tabular}{ll}
\hline Actividades de Viaje & Porcentaje \\
\hline Casa & $2 \%$ \\
Trabajo & $61.7 \%$ \\
Estudios & $4.8 \%$ \\
Compras & $14.2 \%$ \\
Asuntos personales & $13.3 \%$ \\
Otros & $4 \%$ \\
\hline
\end{tabular}

Nota. En la tabla se evidencia que la mayoría de personas se transporta a otros lugares por motivos de trabajo $(61.7 \%)$ y solo un mínimo porcentaje (2\%) permanece en casa sin movilizarse. Fuente: Elaboración propia tomando como base los resultados esta investigación.

Sistema de transporte comercial y particular. De acuerdo con lo que menciona la directora de la ANT del Cañar, en la ciudad de Azogues existen 18 cooperativas de transporte comercial, entre ellos, 6 de taxis y 12 de camionetas; con 399 unidades de camionetas y 162 de taxis respectivamente, cubriendo éstas la zona urbana de la ciudad (Quizhpi, F., comunicación personal, 30 de noviembre de 2020).

Tabla 8

Modo de transporte utilizado

\begin{tabular}{cc}
\hline Transporte & Porcentaje \\
\hline A pie & $8.3 \%$ \\
Bicicleta & $0.7 \%$ \\
Moto & $2.5 \%$ \\
Bus & $7.3 \%$ \\
Automóvil (conduciendo) & $66.7 \%$ \\
Automóvil (acompañante) & $9.2 \%$ \\
Taxi, camioneta de alquiler & $4.3 \%$ \\
Otro & $1 \%$
\end{tabular}

Nota. En esta tabla se evidencia la preferencia del auto particular (66.7\%) y una baja concurrencia con el uso del transporte público. (7.3\%). Estos resultados tienen concordancia con lo expuesto por Pérez Cotrina (2017), en donde los ciudadanos refieren que el transporte público no brinda seguridad y comodidad por lo que prefieran trasladarse en sus vehículos privados. Fuente: Elaboración propia con base en resultados obtenidos de esta investigación.

Sistema Alternativo de Transporte. El transporte alternativo tiene un papel preponderante en algunas ciudades, lo que genera una brecha importante en la lucha contra la contaminación. Hoy en día, debido al covid-19, las restricciones del transporte particular, la limitación de pasajeros en el transporte público, entre otras, han dado lugar al uso de la 
bicicleta básicamente. Así, en ciudades como Quito, han asumido ya, la construcción de ciclovías; en Cuenca, desde el 2019 está en marcha el sistema de bici pública con 240 unidades y 20 paradas, $16 \mathrm{Km}$ de bici-acera y $23 \mathrm{~km}$ de sendas de uso comparativo; y en Guayaquil también impulsaron la Ordenanza para el uso de la bicicleta. (Diario El Comercio, 2020). En el cantón de Azogues no se proponen metodologías específicas para la implementación de espacios a ser usados para la movilidad alternativa, concretamente un sistema de ciclovías.

Tabla 9

Implementación de un Sistema de Préstamo gratuito de Bicicletas

\begin{tabular}{cc}
\hline Opinión de Ciudadanos & Porcentaje \\
\hline Muy buena & $70.8 \%$ \\
Buena & $13.3 \%$ \\
Regular & $9.2 \%$ \\
Mala & $1.3 \%$ \\
No se & $5.4 \%$ \\
\hline
\end{tabular}

Nota. En la tabla se expresa una amplia acogida ante la implementación de un sistema de bicicletas (70.8\%). Fuente: Elaboración propia en base a datos obtenidos de este estudio.

Tabla 10

Posibilidad de uso del Sistema de Préstamo gratuito de Bicicletas

\begin{tabular}{cc}
\hline Uso de Bicicleta & Porcentaje \\
\hline $\mathrm{Si}$ & $67.5 \%$ \\
$\mathrm{No}$ & $8.3 \%$ \\
Tal vez & $24.2 \%$ \\
\hline
\end{tabular}

Nota. La tabla muestra una respuesta afirmativa tras el uso de bicicleta frente a la existencia de un Sistema de Ciclovías. Además, como explica Motos (2019) un indicador, uso de bicicleta, permite cuantificar resultados para la implementación políticas de movilidad sostenible de acorde a las necesidades de una ciudad. Fuente: Elaboración propia en base a los datos que proporciona esta investigación.

\section{Costos}

Tarifas a usuarios. En lo referente al transporte público y sus tarifas, los usuarios cancelan por el servicio, según lo establecido por el Concejo Cantonal desde el año 2016, el valor de $\$ 0.30$ por pasaje completo. Pero, los estudiantes, personas de la tercera edad y personas discapacitadas cancelan $\$ 0.15$, de lo cual los $\$ 0.05$ es subsidiado por el GADMA. Hasta la fecha no se han implementado ningún otro sistema (Pérez Cotrina, 2017, p. 80). En tanto que, el transporte comercial de taxis y camionetas mantienen diferentes tarifas de acuerdo a las distancias, y en caso de las camionetas depende también de la carga que lleven. En la ciudad de Azogues no existe un taxímetro que controle la cantidad a pagar. La tarifa en zona urbana es de $\$ 1,50$ (distancias cortas) pero, desde zonas aledañas (Charasol, Uchupucún, Chacapamba) a la zona central varía hasta $\$ 3$ por viaje (Pérez Cotrina, 2017). 
Tabla 11

Promedio de tarifas de transporte comercial

\begin{tabular}{cc}
\hline Tipo Transporte & Gasto por Semana (\$) \\
\hline Vehículo propio & $* 15.00$ \\
Transporte público. & $* 5.00$ \\
Transporte comercial & $* 3.00$ \\
Otros & $* 5.00$ \\
\hline
\end{tabular}

Contaminación ambiental producida por el transporte. Para analizar las emisiones producidas por el parque automotor, se examina a una cantidad representativa de vehículos, de los cuales la mayoría son a gasolina. Se obtienen 4 grupos: los vehículos livianos a gasolina con inyección $(72,1 \%)$, vehículos livianos a gasolina con carburados $(12,9 \%)$, vehículos pesados a diésel $(6,4 \%)$ y un grupo denominado otros $(8,6 \%)$. De este estudio se obtuvo las emisiones anuales que están entre 15970 toneladas, de las cuales el 94\% representan al CO2 y un 6\% a otros compuestos contaminantes (Peñafiel, 2019).

Ruido. El ruido vehicular es una de las principales fuentes de contaminación humana y ambiental proveniente del transporte circulante, que sucede diariamente como producto de la necesidad de movilizarse de las personas. El ruido está asociado a cuatro fuentes principales: ruido vehicular (de propulsión, de rodadura, aerodinámico, por tráfico vehicular, emisión de bocinas), ruido de propulsión, comportamiento del conductor, construcción y mantenimiento de vías (Ibarra \& Piña, 2011).

Para mejorar la movilidad urbana en el cantón Azogues, es imprescindible contar con una constante actualización de la información, que muestre con datos estadísticos la situación actual de movilidad dentro la misma para pensar en la nueva técnica del urbanismo táctico en las ciudades. Es evidente, en el cantón Azogues no se presentan avances mayormente en temas de sostenibilidad, aún se observa una alta contaminación ambiental, carece de sistemas alternativos de transporte, espacios públicos y zonas peatonales descuidadas.

Po último, el tema del transporte público, debe mejorar para brindar mayor confianza al usuario, y que prioricen el uso de este sistema colectivo de transporte, con lo que se podría mejorar el tema de movilidad en la zona central colapsada por el gran número de vehículos, especialmente en horas pico.

Selección de Indicadores

Para la selección de los indicadores que nos ayudarán a medir la movilidad urbana sostenible en el cantón de Azogues hay que tener en cuenta dos instancias fundamentales: recopilación inicial de indicadores tomados del análisis bibliográfico y la recopilación de indicadores en base al análisis del objeto del presente estudio en Azogues. Después, a partir de la lista general o recopilación inicial, se escogerán los que más se acoplen al estudio bajo algunos criterios:

$\checkmark$ La relevancia otorgada a cada indicador 
$\checkmark$ Disponibilidad de todos los datos

$\checkmark$ Que sean medibles, verificables y cuantificables

$\checkmark$ Comparables, reproducibles y repetibles

$\checkmark$ Que permitan evaluar y medir diversos niveles de sostenibilidad

En base a estos criterios, se elabora un total de 25 indicadores mismos que se clasifican de acuerdo a los tres objetivos principales planteados.

Tabla 12

Mejorar la eficiencia del Sistema de Transporte y Tránsito (objetivo 1)

No. Indicadores Fórmulas

1 Capacidad de servicio transporte público = (CSTP/población total sin vehículo) $* 100$ (CSTP)

2 Velocidad media transporte público terrestre

Distancia total recorrida/Tiempo total del desplazamiento

3 Puntualidad en servicio transporte público

Datos se obtienen directamente

4 Índice de motorización

[No. vehículos registrados a excepción híbridos y eléctricos, en un período de tiempo y en una unidad específica espacial de referencia / No. habitantes que viven dentro de la unidad espacial en cierto de tiempo] ${ }^{*} 1000$

$5 \quad$ Vehículos particulares circulantes (VPC)

(No. VPC en tiempo dado) + (No. motos que circulan en el mismo tiempo)

$6 \quad$ Viajes en transporte no motorizado

(Viajes a pie y en bicicleta/ Total de viajes realizados según encuestas) ${ }^{*} 100$

7 Vehículos que consumen energía renovable (VCER)

(No. VCER matriculados cantón Azogues/ No. vehículos matriculados en Azogues) * 100

8 Tiempo medio en transporte público por trabajo

Sumatoria del tiempo de viajes en transporte público por trabajo/ Población usa transporte público por trabajo

9 Tiempo medio en transporte público por estudio

Sumatoria del tiempo de viajes en transporte público por estudio/Población usa transporte público por estudio

10 Frecuencia de transporte público colectivo

FL1 + FL2 + FLn / Total FLE para área estudio (FLE)

11 Edad del parque automotor transporte público

$\Sigma$ de edad de las unidades que prestan servicio de transporte público colectivo para la zona urbana/Total unidades que prestan servicio transporte público colectivo en zonas urbanas 
12 Calidad de transporte público colectivo

13 Déficit de aparcamiento para vehículo privado (DEFap)

14 Aparcamiento para vehículo privado en la calzada (AVP)

15 Espacio viario para peatones

16 Accesibilidad del espacio de tránsito peatonal

17 Espacio viario para bicicletas

18 Espacio viario para transporte público

19 Vías de acceso adecuadas
[Percepción del estado de la unidad (\% estado bueno) + Percepción del trato al usuario (\% trato bueno) + Percepción de seguridad (\% si es seguro) + Percepción sobre la actitud del conductor (\% de actitud bueno) + Cumplimiento del horario (\% población que dijo que sí cumple) / 5

DEFap $(\%)=[($ Demanda plazas aparcamiento Oferta plazas fuera de calzada) / Demanda plazas aparcamiento] $* 100$

$\operatorname{AVP}(\%)=[$ Plazas de aparcamiento fuera de calzada / Total de plazas de aparcamiento] * 100

$=[$ Longitud de vías adecuadas (estado bueno y material de asfalto o concreto) / Longitud total de vías dentro del área urbana] * 100

[ Longitud del espacio de tránsito peatonal accesible $\geq 1,00 \mathrm{~m}$ / Longitud total del espacio de tránsito peatonal (longitud de acera derecha más longitud de acera izquierda)] * 100

$=$ [Longitud del viario público para bicicletas $/$ (Longitud viario público total) *2] * 100

$=($ Longitud carril bus / Longitud total de vías $)$ * 100

$=[$ Longitud de vías adecuadas (estado bueno y material de asfalto o concreto) / Longitud total de vías que unen el área urbana con las áreas de estudio] * 100

Nota. Esta tabla indica los indicadores correspondientes al primer objetivo de estudio que tiene que ver básicamente con características y elementos del transporte en general. ${ }^{*} \Sigma=$ sumatoria. Fuente: Adaptada de Planificación Estratégica y Construcción de Indicadores (Jiménez, 2008, Diapositiva 33).

Tabla 13

Fortalecer la cohesión Social y Territorial (objetivo 2)

\begin{tabular}{|c|c|c|}
\hline No. & Indicadores & Fórmulas \\
\hline 20 & Costes en transporte público de las familias & $\begin{array}{l}\text { Total de coste del usuario de transporte } \\
\text { público / Total de usuarios de transporte } \\
\text { púbico consultadas que se movilizan }\end{array}$ \\
\hline 21 & Costes en transporte privado de las familias & $\begin{array}{l}\text { Total de coste del usuario de transporte } \\
\text { privado / Total de usuarios de transporte } \\
\text { privado consultados que se movilizan }\end{array}$ \\
\hline 22 & Modos alternativos de transporte. & Los datos se obtienen directamente \\
\hline
\end{tabular}


23 Número de vehículos por familia. Los datos se obtienen directamente

Nota. La tabla muestra los indicadores característicos del objetivo relacionado a la parte social. Fuente: Adaptada de Planificación Estratégica y Construcción de Indicadores (Jiménez, 2008, diapositiva 35).

Tabla 14

Conseguir una movilidad sostenible (objetivo 3)

\begin{tabular}{lll}
\hline No. & Indicadores & Fórmulas \\
\hline $\mathbf{2 4}$ & Nivel de contaminación de los buses & $\begin{array}{l}\text { Considerar las emisiones totales, es decir, las } \\
\text { producidas por vehículos de gasolina, gasóleo y } \\
\end{array}$ \\
& & $\begin{array}{l}\text { gases licuados del petróleo } \\
\text { Valores de distintos rangos indicadores de ruido }\end{array}$ \\
& Población expuesta al ruido procedente del & Valores \\
& transporte & Lden, Ldía, Ltarde y Lnoche se obtienen de la \\
& & suma de los datos de las carreteras.
\end{tabular}

Nota. Fuente: Adaptada de Planificación Estratégica y Construcción de Indicadores (Jiménez, 2008, diapositiva 41).

\section{Aplicación}

Los estudios se aplican a la Ciudad de Azogues, y la situación a medirse es la movilidad sostenible en el área urbana, para ello se considera el Plano de la Ciudad, dividido en zonas, mismo que es tomado de El Plan de Desarrollo y Ordenamiento Territorial del cantón Azogues (FIPAD, 2020). Para el cálculo de los indicadores se toma la información obtenida del análisis bibliográfico, GAD Municipal, ANT y de fuentes primarias, por medio de levantamientos en campo (encuestas).

Tabla 15

Mejorar la eficiencia del Sistema de Transporte y Tránsito en el cantón Azogues (objetivo 1)

\begin{tabular}{|c|c|c|}
\hline No. & Indicadores & Fórmulas \\
\hline 1 & $\begin{array}{l}\text { Capacidad de servicio del transporte } \\
\text { público. }\end{array}$ & $=(1.230 / 2.800) * 100=43.92 \%$ \\
\hline 2 & $\begin{array}{l}\text { Velocidad media del transporte público } \\
\text { terrestre }\end{array}$ & $17.1 \mathrm{Km} / 0,866$ horas $=19.74 \mathrm{Km} / \mathrm{h}$ \\
\hline 3 & $\begin{array}{l}\text { Puntualidad de los servicios de transporte } \\
\text { público }\end{array}$ & $\mathbf{5 3} \%$ opina que no son puntuales. \\
\hline 4 & Índice de motorización & {$[20.849 / 46.787] * 1000=445.61$} \\
\hline 5 & Vehículos privados en circulación & $\begin{array}{l}(88)+(10)=98 \text { por hora no pico en el casco } \\
\text { urbano. }\end{array}$ \\
\hline 6 & Viajes en medios no motorizados & $\begin{array}{l}\text { (Viajes a pie y en bicicleta/ Total de viajes } \\
\text { realizados según encuestas) } * 100\end{array}$ \\
\hline 7 & $\begin{array}{l}\text { Vehículos que consumen energía } \\
\text { renovable }\end{array}$ & $(4.507 / 20.849) * 100=21.61 \%$ \\
\hline 8 & $\begin{array}{l}\text { Tiempo medio en transporte público por } \\
\text { trabajo }\end{array}$ & 45 minutos. \\
\hline 9 & $\begin{array}{l}\text { Tiempo medio en transporte público por } \\
\text { estudio }\end{array}$ & 30 minutos \\
\hline 10 & $\begin{array}{l}\text { Frecuencia de transporte público colectivo } \\
\text { para área estudio (FLE) }\end{array}$ & $14+12+10+12+8+8+10+14 / 8=11$ \\
\hline
\end{tabular}


11 Edad del parque automotor transporte público

12 Calidad de transporte público colectivo

13 Déficit de estacionamiento para vehículo privado (DEFap)

14 Estacionamiento para vehículo privado en la calzada (AVP)

15 Espacio viario para peatones

16 Accesibilidad espacio de tránsito peatonal

17 Espacio viario para bicicletas

18 Espacio viario para transporte público

19 Vías de acceso adecuadas
197/ 41= 4.8 años

$[22+26+15+20+33] / 5=\mathbf{2 3 . 2} \%$

DEFap $(\%)=[(1500-1056) / 1500] \times 100=\mathbf{2 9 . 6 \%}$

$\operatorname{AVP}(\%)=600 / 1056] \times 100=56.81 \%$

$=[3 \mathrm{~km} / 35 \mathrm{Km}] * 100=8.57 \%$

[ $1.15 / 2.5] * 100=46 \%$

$=[0 /(0) * 2] * 100=0$

$=5,8 \%$

$=69 \%$

Nota. Esta tabla muestra lo resultados de diferentes indicadores de transporte y tránsito aplicados en el Cantón Azogues en base a sus necesidades. Fuente: Elaboración propia adaptada de de Planificación Estratégica y Construcción de Indicadores (Jiménez, 2008, diapositiva 33).

Tabla 16

Fortalecer la cohesión Social y Territorial en el cantón Azogues (objetivo 2)

\begin{tabular}{lll}
\hline No. & Indicadores & Fórmulas \\
\hline $\mathbf{2 0}$ & $\begin{array}{l}\text { Costes en transporte público de las } 1.80 / 3=\$ 0.60 \\
\text { familias } \\
\mathbf{2 1}\end{array} \begin{array}{l}\text { Costes en transporte privado de las } 10 / 3=\$ 3.33 \\
\text { familias }\end{array}$ \\
$\mathbf{2 2}$ & $\begin{array}{l}\text { Modos alternativos de transporte. } \\
\mathbf{2 3}\end{array}$ Número de vehículos por familia. & 1
\end{tabular}

Nota. Esta tabla explica lo resultados de diferentes indicadores referentes a la movilidad por parte de familias del Cantón Azogues. Fuente: Elaboración propia adaptada de de Planificación Estratégica y Construcción de Indicadores (Jiménez, 2008, diapositiva 35).

Tabla 17

Conseguir una movilidad sostenible en el cantón Azogues (objetivo 3)

\begin{tabular}{lllll}
\hline No. & Indicadores & Fórmulas & & \\
\hline $\mathbf{2 4}$ & Nivel de contaminación de los buses & $\begin{array}{l}\mathbf{4 5 . 2 5 \%} \text { muy } \\
\text { encuestas) }\end{array}$ & \\
$\mathbf{2 5}$ & $\begin{array}{l}\text { Población expuesta al ruido procedente del } \\
\text { transporte }\end{array}$ & $76 \%$ & & \\
& & & \\
\hline
\end{tabular}

Nota. En esta tabla se manifiesta un alto índice de contaminación en la zona urbana del cantón Azogues. Fuente: Elaboración propia adaptada de Planificación Estratégica y Construcción de Indicadores (Jiménez, 2008, diapositiva 41).

\section{Conclusiones.}

- Con la identificación y elaboración del conjunto de indicadores de movilidad sostenible, se pretende crear un punto de partida para conocer la realidad actual dentro del área urbana de la Ciudad de Azogues y proyectarla a futuro, por lo tanto, el cálculo de indicadores deberá ser constantemente monitoreado con información actualizada, que servirá para la implementación de políticas de planificación en temas de tránsito, transporte, y vialidad. 
- Los indicadores planteados, mantienen una estrecha relación entre sí, pues todos son parte de la movilidad y de la movilidad sostenible, lo que contribuye a su medición y jerarquización, siendo este, otro argumento importante de trabajar con indicadores.

- Considerando el tema de la sostenibilidad, será importante para la Ciudad el control de la contaminación atmosférica, la que debe enfocarse en la eliminación o reducción de gases y partículas que afectan a la salud, así como de ruidos provocados por motores y bocinas, ya que se observa con el $45,25 \%$ o muy contaminante.

- Otro aspecto importante que se aprecia como resultado del estudio, es el incremento del parque automotor en la ciudad de Azogues, lo que ha provocado una inminente congestión vehicular, observando que la mayor parte de la población prefiere movilizarse en vehículos privados, en tanto el transporte público se observa notoriamente disminuido, por lo tanto, éste debería ser mejorado, sus rutas, sus unidades, su servicio; para que las personas opten por utilizar más este medio de transporte dejando de utilizar vehículos particulares, contribuyendo a mejorar la movilidad, así como contaminar menos el ambiente.

- Otro aspecto fundamental para lograr una movilidad sostenible será la incorporación de modos alternativos de transporte, como es el uso de loa bicicleta, lo cual es muy aceptado por la población, se deberá considerar en el estudio vial y urbano, la incorporación de ciclo rutas, zonas de estacionamiento seguro de bicicletas, que en la actualidad no existen para el casco urbano de la Ciudad de Azogues. Por último, se debe actualizar el estudio referido al Servicio Municipal de Estacionamiento Rotativo Tarifado de Azogues SEMERTAZ, pues en la actualidad existe un colapso con estas zonas de parqueo, lo que podría pensar en ampliarse.

\section{Referencias bibliográficas.}

Agencia Nacional de Tránsito. (2017). Número de vehículos matriculados en la ciudad de Azogues año 2017. https://www.ant.gob.ec/index.php/noticias/487-el-81-devehiculos-matriculados-en-canar\#.YA6O5uhKjIU

Alarcón, J. (2020). La ciudad compacta y la ciudad dispersa: Un enfoque desde las perspectivas de convivencia y sostenibilidad. Revista San Gregorio, (39), 1-14. http://dx.doi.org/10.36097/rsan.v1i39.1411

Caniffi, S. (23 de marzo de 2017). Cinco observaciones a la medición de la movilidad sostenible [Publicación de blog]. Geo innova. https://geoinnova.org/blogterritorio/medicion-de-la-movilidad-sostenible/\# marzo, 23, 2017

Centro de Transporte Sustentable EMBARQ (2015). Indicadores para el ejercicio del derecho a la movilidad. http://centro.paot.org.mx/documentos/paot/estudios/PAOT_CTSEMBARQMexi co.pdf 
Diario El Comercio (06 de junio de 2020). La pandemia abrió campo a la movilidad sostenible en bicicleta. $\quad$ El Comercio. https://www.elcomercio.com/actualidad/pandemia-movilidad-sosteniblebicicleta.html

Díaz-Contreras, C., \& Díaz-Vidal, G. (2019). ¿Hacia un planeta sostenible?. Revista chilena de ingeniería, 27(2), 185-187. https://scielo.conicyt.cl/pdf/ingeniare/v27n2/0718-3305-ingeniare-27-0200185.pdf

Dirección General de Industria, Energía y Minas de la Comunidad de Madrid (2010). Movilidad Urbana Sostenible: Un reto energético y ambiental. http://www.upv.es/contenidos/CAMUNISO/info/U0536159.pdf

Ecologistas en Acción. (16 de noviembre de 2017). Problemas de la movilidad y su repercusión urbana e individual [Publicación de blog]. https://www.ecologistasenaccion.org/9845/problemas-de-la-movilidad-y-surepercusion-urbana-e-individual/

Espejel, I. (2009). Modelo de la OCDE para medir el desarrollo sustentable: ejemplos [Archivo

$\mathrm{PDF}]$. http://www.iiec.unam.mx/sites/www.iiec.unam.mx/files/Lecturas_ponencias/Ilea naEspejel.pdf

Falcón Suárez, H., Verano Tacoronte, D., \& García Santana, A. (2016). La movilidad urbana sostenible y su incidencia en el desarrollo turístico. Gestión y Ambiente, 19(1), 48-62. https://revistas.unal.edu.co/index.php/gestion/article/view/57205/56834

Flores Juca, E., García Navarro, J., Chica Carmona, J., \& Mora Arias, E. (2017). Identificación y análisis de indicadores de sostenibilidad para la movilidad. Revista de la Facultad de Arquitectura y Urbanismo de la Universidad de Cuenca, 6(11), 99-100. https://doi.org/10.18537/est.v006.n011.a07

FIPAD. (2020). Plan de Desarrollo y Ordenamiento Territorial del Cantón Azogues. Gobierno Autónomo Descentralizado del Municipio de Azogues.

Gakenheimer, R. (1998). Los problemas de la movilidad en el mundo en desarrollo. Revista Latinoamericana de Estudios Urbano Regionales, 24(72), 33-52.

http://dx.doi.org/10.4067/S0250-71611998007200002

García Schilardi, M. (2017). Evaluación de la dimensión operativa del transporte colectivo en el área metropolitana de Mendoza, Argentina. Revista Perspectiva Geográfica, 22(2). https://doi.org/10.19053/01233769.5965

Gaviria, P. (2013). Diseño de un sistema de indicadores de sostenibilidad como herramienta en la toma de decisiones para la gestión de proyectos de infraestructura en Colombia [Tesis de Maestría, Universidad de EAFIT]. 
Repositorio Institucional

EAFIT. https://repository.eafit.edu.co/handle/10784/1250

Gobierno Autónomo Descentralizado del Municipio de Azogues. (2013). Plan de Transporte, Tránsito y Seguridad Vial de Azogues-Cañar. http://www.azogues.gob.ec/portal/index.php/resoluciones

Guillamón, D. \& Hoyos, D. (Ed.). (2005). Movilidad Sostenible: de la teoría a la práctica. Zaindu Gure Etxea. https://www.mrafundazioa.eus/es/centro-dedocumentacion/medioambiente/inguru-gaiak-movilidad-sostenible-de-la-teoriaa-la-practica

Herrera, F. \& Jaramillo, C. (20 de diciembre de 2006). Los polos generadores de viajes en Santiago de Cali. Monografías.com. https://www.monografias.com/trabajos42/viajes-santiago-cali/viajes-santiagocali2.shtml

Ibarra, M., \& Piña, J. (2011). Propuesta para el mejoramiento del transporte público urbano para la ciudad de Azogues con perspectivas hacia: la seguridad vehicular, contaminación ambiental y gestión del tránsito [Tesis de fin de Grado, Universidad Politécnica Salesiana de Cuenca]. Repositorio - Institucional Universidad Politécnica Salesiana de Cuenca. https://dspace.ups.edu.ec/handle/123456789/1116

Instituto Nacional de Estadísticas y Censos. (2010). Base de datos-censo de Población y Vivienda 2010. https://www.ecuadorencifras.gob.ec/base-de-datos-censo-depoblacion-y-vivienda-2010/

Jiménez, R. (2008). Planificación Estratégica y Construcción de Indicadores en el Sector Público de Costa Rica "Metodología para la Construcción de Indicadores [Diapositiva de PowerPoint]. CEPAL. https://www.cepal.org/ilpes/noticias/paginas/4/34184/PresentacionIndicadores.p df

Lizárraga, C. (2006). Movilidad urbana sostenible: Un reto para las ciudades del siglo XXI. Economía, Sociedad y Territorio, 6(22), 1-35. https://est.cmq.edu.mx/index.php/est/article/view/260/265

Mendoza, M. (31 de enero de 2017). ¿Qué es Movilidad Urbana? [Publicación de blog]. Un Lugar. https://unlugar.org.mx/que-es-movilidad-urbana/

Ministerio de Transporte de Obras Públicas. (2020). Política Nacional de Movilidad Urbana Sostenible. https://www.obraspublicas.gob.ec/politica-nacional-demovilidad-urbana-sostenible-2/

Miralles-Guasch, C., \& Cebollada, A. (2009). Movilidad cotidiana y sostenibilidad: una interpretación desde la geografía humana. Boletín de la Asociación de Geógrafos Españoles,

193-216. https://www.researchgate.net/publication/40700310_Movilidad_cotidiana_y_sos tenibilidad_una_interpretacion_desde_la_geografia_humana 
Morocho, A., \& Rodríguez, J. (2019). La calidad de servicio del transporte público urbano en la ciudad de Azogues [Tesis de fin de Grado, Universidad Politécnica Salesiana de Cuenca]. Repositorio - Institucional Universidad Politécnica Salesiana de Cuenca. https://dsde pace.ups.edu.ec/bitstream/123456789/17313/1/UPSCT008257.pdf

Motos, G. (2019). Análisis de indicadores de movilidad urbana sostenible [Tesis de Maestría, Universidad Politécnica de Cartagena]. Repositorio Institucional Universidad Politécnica de Cartagena. https://repositorio.upct.es/bitstream/handle/10317/7784/tfm-motana.pdf? sequence $=1 \&$ is Allowed $=y$

Ordenanza Sustitutiva de Reglamentación del Uso de Suelos Urbano y Rural de Azogues. Concejo Municipal de Azogues, 77, 5 de mayo de 2009. http://www.azogues.gob.ec/portal/index.php/ordenanzas/category/72ordenanzas-municipales

Peñafiel, M. (2019). Estimación de un Inventario de Emisiones de Fuentes Móviles Terrestres para la Ciudad de Azogues Aplicando El Modelo Internacional de Emisiones Vehiculares [Tesis de Maestría, Universidad del Azuay]. Repositorio - Universidad del Azuay. http://dspace.uazuay.edu.ec/handle/datos/8894

Pérez Cotrina, D. (2017). Estudio de calidad de suelos con fines de cimentación asociación pro - vivienda el Eden 2 - Pimentel [Tesis de fin de Grado, Universidad César Vallejo]. Repositoprio Institucional - Universidad César Vallejo. https://repositorio.ucv.edu.pe/bitstream/handle/20.500.12692/16960/perez_cd.pd f? sequence $=1$.

Quiroga, R. (2009). Guía metodológica para desarrollar indicadores ambientales y de desarrollo sostenible. Comisión económica para América Latina y el Caribe, (61), 1-129. https://repositorio.cepal.org/handle/11362/5502

Rodríguez-Chávez, H. (2016). Indicadores cualitativos y estrategias para una movilidad sostenible en la ciudad de Hermosillo, Sonora (México) [Tesis de fin de Grado, Universidad Politécnica de Catalunya]. Repositorio Institucional - Universidad Politécnica de Catalunya. https://upcommons.upc.edu/bitstream/handle/2117/100163/TFMHector\%20Rodriguez.pdf?sequence=1 yisAllowed $=\mathrm{y}$

Torres, J. D. (2015). Estudio de Viabilidad en la implementación de vehículos eléctricos en la ciudad de Cuenca [Tesis de fin de Grado, Universidad Politécnica Salesiana de Cuenca]. Repositorio Institucional - Universidad Politécnica Salesiana de Cuenca. https://dspace.ups.edu.ec/bitstream/123456789/8050/1/UPSCT004893.pdf 
Universidad Técnica de Valencia. (2012). Movilidad y Transporte. Texto de Red Asturiana http://www.upv.es/contenidos/CAMUNISO/info/820042normalc.html

Urbano, P., Ruíz, A., \& Sánchez, J. I. (2016). Indicadores de sostenibilidad para el transporte. Una perspectiva estructural. Reunión de Economía Mundial (Informe 13). http://xiiirem.ehu.es/entry/content/237/cod_049.pdf

Zuluaga, J. F. (22 de octube de 2017). ¿Qué es movilidad sostenible y por qué es importante? [Publicación de blog]. Sura. https://blog.segurossura.com.co/articulo/movilidad/por-que-debes-saber-que-esmovilidad-sostenible 


\section{PARA CITAR EL ARTÍCULO INDEXADO.}

León Andrade, M. P., \& Carriel Bustamante, V. V. (2021). Indicadores para medir la movilidad sostenible en espacios urbanos de la sierra ecuatoriana. Caso de estudio el cantón Azogues. ConcienciaDigital, 4(1.2), 489-515. https://doi.org/10.33262/concienciadigital.v4i1.2.1738

\section{Ciencia
Digital
Ethtoul}

El artículo que se publica es de exclusiva responsabilidad de los autores y no necesariamente reflejan el pensamiento de la Revista Conciencia Digital.

El artículo queda en propiedad de la revista y, por tanto, su publicación parcial y/o total en otro medio tiene que ser autorizado por el director de la Revista Conciencia Digital.

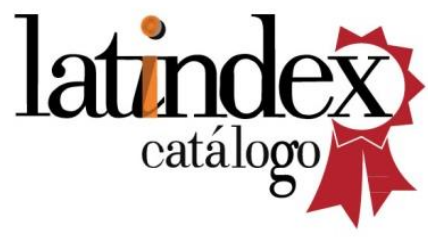

